NASA Technical Memorandum 103135

AIAA-90-1994

\title{
Digital Filtering of Plume Emission Spectra
}

George C. Madzsar

Lewis Research Center

Cleveland, Ohio

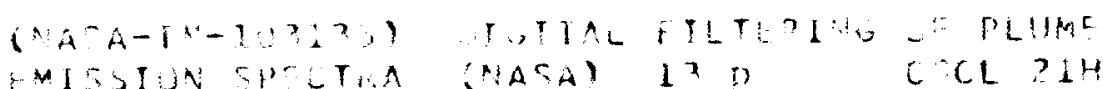

$$
\begin{aligned}
& 1,20-24373 \\
& \text { unclus } \\
& 93 /<0 \quad 0275241
\end{aligned}
$$

Prepared for the 26th Joint Propulsion Conference cosponsored by the AIAA, ASME, SAE, and ASEE Orlando, Florida, July 16-18, 1990 



\title{
DIGITAL FILTERING OF PLUME EMISSION SPECTRA
}

\author{
George C. Madzsar \\ Vational teronautics and Space Administration \\ Lewis Research Center \\ Cleveland, Ohio 44135
}

\section{Abstract}

Fourier transformation and digital filtering techniques were used to separate the superpositioned spectral phenomena observed in the exhaust plumes of liquid propellant rocket engines. Space shuttle main engine (SSME) spectral data were used to show that extraction of spectral lines in the spatial frequency domain does not introduce error, and extraction of the background continum introduces only minimal error. Error introduced during band extraction could not be quantified due to poor spectrometer resolution. Based on the atomic and molecular species found in the SSME plume, it was determined that spertrometer resolution must be $0.03 \mathrm{~nm}$ for SSME plume spectral monitoring.

\section{Int roduction}

Spectral analysis of the radiation emitted from the exhaust plume of an oxygen - hydrogen $(\mathrm{OH})$ fueled rocket engine reveals the constituents $1-5$ and the state(s) $)^{6-8}$ of the exhatst gases. Engine health and performance characteristics can be inferred from knowledge of the exhaust constituents and states.

An inherent assumption with plume spectral analysis is that the spectral characteristic(s) identifying a particular constituent or state can be distinguished from all other spectral phenomena occurring simultaneously within the plume. This assumption is not always correcl. A high resolution spectrometer can separate closely spaced spectral lines, however, it cannot separcte superpositioned spectral phenomena. Since the rverall irradiance from a plume is the sum of incariances from numerous discrete spectral sources, superpositioning does occur, thereby preventing direct determination of the absolute irradiances from discrete spectral phenomena.

This paper demonstrates the application of Fourier and digital filtering techniques to the analysis of rocket engine plume spectra. These tools offer the capability to d ssect a spectrum and examine its specific features in detail.

\section{Background}

By monitoring the contents of the rocket engine exhaust, it is possible o determine which material(s), and therefore which components within the engine, are eroding. Figur, 1 displays the propellant flow paths of the space shuttle main engine (SSME). All particulates eroding from internal engine components within the flow path end up in the combustion chamber where they burn and exit through the exhaust nozzle. Knowledge about the state of the exhaust gases is bineficial for evalua$t i$ on of the combustion process. Spectral emissions from the by-products of $\mathrm{OH}$ basel combustion can provide information about propellant mixture ratio, and the blackbody radiation from particulates can provide information about the temperature in the combust ion chamber.

Copyright (C) 1990 by the American Institute of Aemautics and Astronautics, Inc. No copyright is assertec in the United States under Title 17, U.S. Code. The U.S. Government has a royalty-free license to exercise all rights under the copyright claimed herein for Governmental purposes.

All other rights are reserved by the copyright as ner.
Table 1 is a partial list of SSME materials and their corresponding emission wavelengths. The wavelength values are based on past SSME firings. 1 and predicted values. 9,10 ds of this writing, the spectral phenomena of interest lie between the far ultraviolet and near infrared wavelengths. Observing spectral irradiance at wavelengths which correspond to engine structural materials as a function of time identifies both the species and relative rates of material erosion. Based upon the state of the exhaust gases and the species and rate of the material erosion. relative health and performance of the rocket engine can be determined.

Figures 2 to 4 display typical emission spectra from the SSUE. As evident from Fig. 2, line emissions are superimposed on band emissions, which are superimposed on a background continuum. A series of emission spectra recorded as a function of time from an SSME are displayed in Fig. 3 . The $x, y$ and $z$ axes correspond to wavelength, irradiance, and time respectively. The initial spectrun was recorded just after engine ignition, and subsequent spectra were recorded at 0.1 sec intervals. As can be seen, irradiance levels change substantially over time.

The current technique used to analyze SSME, spectral data consists of tracking irradiance levels at wavelengths corresponding to specific engine materials, without considering the effects of spec$t$ ral superpositioning. ${ }^{1-4}$ This method is acceptable if a proportionality constant between the irradiances of the various spectral phenomena exists. and does not change with engine operating conditions. This proportionality constant is dependent on the amount and species of impurities in the propellants. the rate of material erosion from engine components. propellant mixture ratio. combustion chamber pressure and temperature, and propellant flow rates. From the spectral diversity seen in Figs. 2 to 4 . it may be difficult to determine if a proportionality constant even exists. In Fig. 3. the continumm and spectral line irradiances vary with respect to time (corresponding to power level changes), and vary with respect to each other. [n Fig. 1, spectral line and continum irradiances appear substantially different than the corresponding irradiances seen in Figs. 2 and 3 . Considering these variations, it could not, for example, be determined if a change in the irradiance at a wavelength corresponding to a metal is due to a change in the rate of metal erosion, due to a change in the continuum onto which the metallic spectral line is superimposed, or due to changes in both the continum and the rate of metal erosion. Using simple background subtraction to isolate background changes from line changes is not ideal since with background subtraction, absolute irradiance of the spectral line may be lost. Also, background subtraction cannot separate line from band emissions.

Note that the spectra displayed in Figs. 2 to 4 were selected to demonstrate the diversity of SSTIE plume emissions. From these figures, the following inferences can be made: First, it may be difficult 
to establish a baseline spectral signature due to gross differences in the spectra from different engines. Second, the use of narrow band pass optic filters to view a few specific wavelengths without regard to irradiances at neighboring wavelengths is precluded.

\section{Theory}

To relate Fourier and digital filtering techniques to plume spectral analysis, consideration must be given to the physics of spectral emissions, and the mathematics of Fourier analysis. The interactions between the sources of plune emissions. causes for variations in plume emissions, Fourier theory, filter implementation, and errors associated with digital filtering need to be understood. Following is a discussion on these issues.

Spectral radiation is the result of photon emission during the combustion of propellants, the burning of impurities in the propellants, and the burning of materials that may have eroded from internal engine components. Energy released during the combustion process excites the atomic, molecular and chemical species within the combustion chamber, which in turn, emit photons. Photon wavelength(s) are a characteristic of their source. Potential types of emissions and sources of photons include the following: 11,12

(1) Line emission from atomic transitions due to thermal excitation.

(2) Line emission from atomic transitions excited by chemical reactions.

(3) Band emission from thermally excited molecules.

(4) Band emission from chemically excited molecules.

(5) Continuum radiation from chemical reactions.

(6) Continuum blackbody radiation from particulates.

Line and bandwidths of these emissions are as their names imply; line emission appears as narrow spectral lines, band emission consists of spectral emissions distributed over a finite band of wavelengths, and the continuum covers the entire spectrum in the observed wavelength range.

In optically thin gases, the shapes of the spectral lines are determined primarily by the velocity and collisional behavior of the emitting species. Factors contributing to variations in the lineshape include the following: 11

(1) Doppler broadening - due to relative motion between atoms and the observer.

(2) Field broadening - result of electrical (Stark effect) or magnetic (Zeeman effect) fields.

(3) Holtsmark broadening - result of collisions between like atoms.

(4) Lorentz broadening - results from the collision of unlike atoms.
(5) Natural broadening - linked to the radiative lifetime through the Heisenberg uncertainty principle.

In an $\mathrm{OH}$ rocket engine plume, the population of metallic species is relatively small compared to the population of combustion species, resulting in minimal Holtsmark broadening of metallic lines. Field broadening is minimal since external magnetic and/or electric fields are not applied. Natural linewidths of atomic emission are on the order of $10^{-6} \mathrm{~nm}$ and the Lorentz and Doppler linewidths are on the order of $10^{-3} \mathrm{~nm}$. Therefore, only Doppler and Lorentz broadening need to be considered for line emissions. Broadening does not need to be considered for the continum and band emissions since broadening induced width variations are small when compared with the natural widths of these phenomena. These differences in line/band widths are the key feature that enable fourier techniques to separate spectral phenomena.

Fourier techniques enable separation of spectral phenomena since different types of spectral emissions have different bandwidths, and therefore different spatial frequencies. The continuum, with a bandwidth covering the entire spectrum, has a relatively low spatial frequency. Line emissions have high spatial frequencies, and band emissions have spatial frequency components greater than that of the continuum, and less than that of the lines.

The Fourier transform is a mathematical tool that relates time or space domain data to its corresponding frequency (or spatial frequency) representation. $3-16$ The Fast Fourier Transform (FFT) is an efficient computer implementation of the Fourier transform. Hith respect to spectroscopy, the space domain of a spectrum is transformed into its spatial frequency counterpart. For spectroscopic applications, the aspects of Fourier theory that must be considered are the spatial sampling interval. issues of data nonperiodicity, and the number of spectral data points to be transformed.

The spatial sampling interval is defined as $d x$, where $d x$ is the resolution of the spectrometer. The Nyquist limit, defined as $1 /(2 \mathrm{dx})$, is the greatest frequency that can be resolved without aliasing errors being introduced into the data set. Therefore, an emission spectrum with sampled data is bandwidth limited to spatial frequencies less than or equal to one-half of the spatia! sampling rate. With respect to spectroscopy, two factors must be considered when determining the required spatial sampling rate. The spatial sampling rate must be adequate to resolve closely spaced spectral lines, and adequate to enable separation of the line, band and continuum spatial frequency components .

A potential error in Fourier transformed data can be caused by the nonperiodicity of the data set that is being transformed, and the assumption that the total duration of the response is the same as the period of the data set. 14 If a data stream is nonperiodic (as are plume spectra), the periodicity assumptions will result in "pollution" of the transformed data set at its two ends. This wraparound characteristic, which is inherent to the FFT, may be overcome by creating a buffer zone containing zeroes at the two ends of the data stream prior to transformation. The number of padding values added at each end of the data stream is equal to half of 
the total number of data points $n$ the unpadded set. The resultant padded set contains twice the number of data points as in the uriginal unpadded data set, while maintaining the power of two data points that are required for the FFT.

If the data set is offset, hat is the first and last values of the data set are not equal to zero, zero padding will introduce vertical edges. Vêrtical edges will exhibit the characteristic $\sin (x) / x$, or damped "ringing" at frequencies corresponding to the edges when the data set is transformed. This may be avoided by subtracting the values of the straight line connecting the first and last points from the entire lata set, and then padding with zeroes. However, the absolute irradiance levels of the original data will then be lost. An alternate method is to pad the left side of the data set with the value of the $f$ rst data point of the original unpadded set, and to pad the right side of the data set with the value o! the last data point of the original unpadded sut

Filtering in the frequency clomain is performed by removing the spatial frequency components corresponding to unwanted spectral phinomena. For filtering, the spectrum is sampled, digitized, padded. and transformed into its frequency representation. The transformed data is multiplied by a filter function which removes all unwanted spatial frequency components. The resultant data s transformed back into its original domain via the inverse FFT. d low pass filter extracts the spatial frequency components of the continuum, a band piss filter extracts the components of the band emiss:ons, and a high pass filter extracts the components of the line emissions. Key issues with this method of filtering are the filter cutof spatia: frequencies, and the filter function shape.

The cutoff spatial frequency defines the boundary between the filtered and unfiltered regimes on the $t$ ransformed spectrum. This cutoff can be determined analytically from the theoretical widths of spectral phenomena, Line emissions have widths on the order of $10^{-3} \mathrm{~nm}$ after broadening. Since spatial frequency is defined as $1 /$ bandwidth, line emissions have spatial frequencies on the order of $1000 \mathrm{cycles} / \mathrm{nm}$. Band emissions lypically have bandwidths on the order of $10 \mathrm{~nm}$, corresponding to spatial frequencies of 0.1 cycles: $/ \mathrm{nm}$. Setting the cutof spatial frequency of the filter between these values will separate them.

The $r i s i n g$ and/or falling eriges of the filter cutoff should not be vertical, since these edges will be transferred to the data set upon application of the filter. Upon inverse transformation, any vertical edge will appear as a $\sin (x) / x$ function in the filtered spectrum. A Gaussian, sine or cosine function may be used to develop the rising and falling edges of the filter.

Note that individual classes of emissions (line, band, continuum) contain spatial frequency components in the other classes, since spectral emissions typically cannot be expressed as a perfect sinusoidal waveform of a sirgle spatial frequency. Therefore, filtering for a specific class of emissions introduces errors. This error is relatively small if the magnitudes of the spatial frequency components outside of its class are small, and can be minimized by careful selection of cutof spatial frequencies.

\section{Experimental Implementation}

Plune emission spectra can be measured with equipment similar to that shown in Fig. 5 . Radiation emitted from the shock diamond within the plume is focused on to a bundle of optical fibers, which trinsmit the light to a spectrometer. The shock diamond serves as the focal point since plume irradiance is greatest at that location. Optical fibers are used since they enable the delicate spectrometer to be placed away from the harsh engine environment. The spectrometer separates the light into its constituent wavelengths. This spectrum is imaged outo a linear photo diode array, which meas ures the irradiance as a function of wavelength. All plume spectra displayed in this paper were recorded at the NASA Stennis Space Center from an SSME during test firing.

The spectra shown in this paper were measured with a 236 element diode array on a $0.5 \mathrm{~m}$ focal length sjectrometer, with a nonrotating 300 groves per millimeter grating. This arrangement yields a wavelength range of 300 to $825 \mathrm{~nm}$, a resolution of $(825-300) / 256 \simeq 2.1 \mathrm{~nm}$, and a vyquist limit of 0.25 cycles $/ \mathrm{nm}$. The resolution, limited primarily by the diode array density, is poor, and does not allow for accurate measurements of closely spaced or narro* spectral lines. With the Stennis equipment, $\left[w_{1}\right]$ complete spectral scans per second can be measured, digitized and transferced to a storage medi um.

Figure 6 displays the spectrum from Fig. after $t r$ insformation, along with superimposed high. low and jand pass filter functions. The upper spatial fretuency in Fig. 6 corcesponds to the Kyquist limit. Vote that the magnitude of the transformed spectrum is plotted in a logarithmic scale to graphically emphasize small changes, and the filter functions ar? plotted in a dimensionless absolute linear scale extending from 0.0 to 1.0 .

Selection of cutoff frequencies for the various filtars was not simple due to poor spectrometer resolution. The spectrometer, with a resolution of $2.13 \mathrm{~nm}$, measured irradiances of spectra! lines with widths of $10^{-3} \mathrm{~nm}$. This resulted in measured line widths of the same order-of-magnitude as the actual widths of the bands. Upon transformation, line and band spatial frequency components overlapped.

Therefore, the cutof frequencies were determined by trial and error. The cutoff frequencies were as follows: 0.025 cycles/nm for the low pass filter. 0.025 cyiles/nm for the high pass filter, and 0.010 through 0.210 cycles/nm for the band pass filter.

\section{Results}

The results of low pass. high pass, and band pass filtering can be seen in Figs. 7 to 9 respectively. In Fig. 7, two curves are displayed. The dotted line is the unfiltered spectrum, and the solid line is the spectrum after low pass filtering. Figures 3 and 9 display the filtered spectrum along with the noise floor. Analysis indicated that low and high pass filtering introduce only minimal error. Band pass filtering does not clearly indicate the capability to extract band emissions. It was concluded that poor spectrometer resolution caused this problem. Poor resolution also caused difficulties with the separation of closely spaced spectral lines. Electrical and/or optical noise was relatively low, indicating a high signal to 
noise ratio. Following is a detailed discussion of these results.

By comparing the unfiltered to the low pass filtered spectrum in Fig. 7, it can be seen that the higher spatial frequency line and band components were removed, as expected. Also from Fig. 7 the reasonableness of the low pass filter can readily be seen, since the filtered spectrum is clearly a smooth fit to the unfiltered spectrum.

The continuum and most lower spatial frequency band emissions were removed by the high pass filter, as seen in Fig. 8. To check the validity of the filtered spectra, irradiance of the sodium line at $589 \mathrm{~nm}$ was examined. First, sodium ir radiance was determined by subtracting the low pass filtered spectra from the unfiltered spectra. The irradiance value at $589 \mathrm{~nm}$ from this subtraction was compared with that from the high pass filtered spectra. The difference between these values was approximately 8 percent, indicating good correlation. In Fig. 8 some lines have multiple species identification labels on them. This resulted from poor spectrometer resolution, which did not enable resolution of closely spaced lines.

[t is evident from Fig. 8 that irradiances after filtering can be extremely small, raising concerns with the signal to noise ratio. Typically, system noise is determined from the dark current that is measured by the individual diodes, and signal to noise is determined by comparing the dark current values to the spectral irradiance values. For this paper the actual signal to noise ratio could not be determined since the dark current scans were not available from Stennis. To perform noise analysis, the dark current value as specified by the diode manufacturer was used. These specifications stated that the maximum dark current noise for any diode is 1 percent of the saturated signal. Therefore, assuming that the peak measured irradiances are near the saturation point, the maximum noise level can be determined. The dashed line in Fig. 8 indicates this 1 percent noise floor.

With Fourier analysis, averaging improves the signal to noise ratio. For emission spectroscopy however, averaging prevents observation of sudden i rradiance changes indicative of engine degradation. For spectroscopic applications, signal to noise is optimized through selection of the diode integration time. The integration time must be lang enough to insure that the measured irradiances are substantially above the detector dark current, yet short enough to prevent diode saturation, and short enough to enable processing of sudden irradiance changes. The overall irradiance from the SSME plume is sufficiently large so long integration times are not required.

Interesting observations can be made from the extracted phenomena seen in Fig. 8. First, the majority of lines observed in Fig. 8 can be identified from Table 1 . Second, noise does not appear to be a problem. Third, it is evident that a great deal of spectral activity is occurring within the plume. The lines corresponding to sodium and potassium are clearly visible. These elements are introduced as impurities in the propellants. Excluding these lines, it is evident that many structural elements are burning. The chromium, copper, iron, nickel, and cobalt lines are present, indicating material erosion. Lithium and calcium lines are present, indicating bearing wear. Typically, all components within the flow path of the engine erode to some degree. However, a sudden change in the amount of erosion could indicate accelerated erosion and/or impending component failure.

Figure 9 displays the spectrum shown in Fig. 4 after band pass filtering, with the dashed line indicating the 1 percent noise floor. As can be seen, band emissions are not accurately extracted. While some bands can be identified, it appears that many unknown artifacts are present. This was due to a number of reasons. First, band emissions were not prominent in the unfiltered spectrum. as evident from Table 1 and Fig. $t$. Second, poor spectrometer resolution did not enable separation of the line and band spatial frequency components. And third, some spatial frequency components of the bands were lost during filtering. It appears that the second and third causes are the predominant drivers in the inability to extract band emissions.

Poor spectrometer resolution causes line emissions to appear much broader than they actually are A resolution of $2.1 \mathrm{nn}$ implies that the reasured wing of a line is at least $2.1 \mathrm{~nm}$ wide, and that the narrowest measurable line width is $4.2 \mathrm{~nm}$. This causes difficulties with spatial frequency overlap. since widths of the artificially broadened lines as measured by a spectrometer are in the same order-ofmagnitude as the actual widths of the bands. This overlap precludes the formation of a distinct boundary between the frequency components of the lines and bands. With no distinct boundary, a cut off spatial frequency for the filter cannot be determined.

Since fourier transformation converts the spec tra into a series of sinusoidal components. the decomposition of a specific type of spectral emis sion will typically be expressed over a range of spatial frequencies rather than at a single spatial frequency. This could translate into a specific type of emissions (line, band, and continuwn) having sine components over a broad range of spatial frequencies, some of which may overlap with the sine components of the other types of emissions. If the magnitudes of the overlapping components from one type of emission are large with respect to the magnitudes of the other type of emission. filtering will corrupt the data.

From Fig. 4 and 7 it can be seen that line emissions occur at high spatial frequencies only. Therefore, removing the lower spatial frequency components with a high pass filter does not introduce error. While the continuum consists mainly of low spatial frequency components, it may have some components at the higher frequencies, which will be filtered out by a low pass filter. From Fig. 5 it can be seen that magnitudes at the low spatial frequencies are substantially greater than magnitudes at all other spatial frequencies, indicating that higher frequency components of the continuum are negligible. Therefore, low pass filtering introduces only minimal error. The magnitudes at spatial frequencies corresponding to the band emissions are relatively small, but could not be distinguished from continuum and line frequency components. Since the bands could not be accurately extracted, errors associated with this process could not be quant if ied. 
A conclusion from the preceding discussion is that line versus band characteristics drive the spectrometer resolution requirements. However, the resolution of the spectrometer must be adequate to separate closely spaced lines as well as separate the line/band spatial frequencius. From Table 1 , nearest lines from different metallic species are the copper line at $353.04 \mathrm{~nm}$, and the cobalt line at $352.98 \mathrm{~nm}$. Assuming these are the nearest lines that must be resolved, the requ: red resolution can be determined. The numeric diflerence between these lines is $0.06 \mathrm{~nm}$. With respect to the Nyquist criteria, the spectrometer must therefore be capable of resolving $0.03 \mathrm{~nm}$ to separate these lines. With this resolution, the measured line widths will be greater than the actual line widths after broadening, indicating that broadening induced line overlap will not be of concern. This resolution corresponds to a spatial frequency of 33.33 cycles $/ \mathrm{nm}$, which is two orders-of-magnitude greater than the spatial frequencies of the band emissions. Therefore, this resolution is sufficient to separate lines and bands, as well as resolve closey spaced lines.

The spectrum shown in Fig. \& was recorded from engine number 2031 during firing number 904-047, on August 11,1989 . This particular spectrum corresponds to emissions occurring 3 sec after engine ignition. Analys is of the 100 sequential scans recorded during the first 50 se: of firing time from this engine using the techuiques discussed in this paper revealed no anomalous engine behavior or accelecated material erosion. Viewing the unfiltered emission spectra as a funition of time did reveal changing irradiance levels. However, upon filtering, irradiances of the soectral lines corresponding to metallic species we:e constant with respect to engine power level, indicating normal engine wear. The same conclusion was reached during post firing analysis of the engine.

\section{Conclusions}

Fourier and digital filtering techniques enable separation and extraction of superpositioned spectral phenomena observed in the $\mathrm{Jlumes}$ of $\mathrm{OH}$ fueled rocket engines. Fourier transformation separates spectral phenomena as a function of spatial frequency, and filtering in the spatial frequency domain extracts the specific spectral phenomena.

In the spatial frequency domain, spectral line emissions appear as the high spitial frequency components, the continuum appears as the low spatial frequency components, and the band emissions appear at spatial frequencies between the 1 ine and continuum components. A high pass filter extracts the line emissions, a low pass filtar extracts the continuum, and a band pass filter extracts the band emissions. Cutoff frequencies for the filter functions can be estimated analytically from the theoretical widths of the various spectral phenomena. To utilize these theoretical cutof $\mathrm{f}$ values, resolu$t i$ on of the spectrometer must be adequate to enable resolution of the spatial frequencies in the range of the calculated cutof $f$ values

High pass filtering does not introduce error since line emissions consist of high spatial frequency components only. Low pass filtering introduces minimal error since the continuum contains some low magnitude higher spatial frequency components which are filtered out. Error introduced by band pass filtering could not be quant ified, since band emissions contain frequency components across much of the spatial frequency spectrum, and the spatial frequencies of the lines and band overlapped. These difficulties with band extraction can be eliminated *ith better spectrometer resolution.

Specific types of emissions (I ine, band, and continutum typically are expressed over a range of spatial frequencies rather than at a single spatial frequency. Therefore, frequency components of the specific types of emissions may overlap. If the magnitudes of these overlapping components are large. filtering will cause corruption in the extracted phenomena

Poor spectrometer resolution causes difficulties with spectral analysis. Poor resolution prevents isolation of closely spaced spectral lines, causes the lines to appear much broader than they actually are, and results in the inability to separate the spatial frequency components of line and band emissions. It was determined that the required resolution for SSHE plume spectral monitoring is $0.03 \mathrm{~nm}$

For the spectra analyzed in this paper. spectromeler resolution was not adequate to separate closely spaced lines or separate the spatial frequency components of the lines and bands. Therefore, the cutoff spatial frequencies for the filters were determined by trial and error. the cutoff frequencies were: $0.025 \mathrm{cycles} / \mathrm{nm}$ for the low pass filter. 0.025 cycles/nm for the high pass filter. and 0.010 through 0.210 cycles/nn for the band pass filter.

As an example of digital filtering implementation. the 100 spectral scans recorded from engine number 2031 during test number $90+-047$ on dugust 11 , 1989 at the VASA Stennis Space Center were analyzed using the techniques discussed in this paper. This analysis revealed no anomalous conditions in the engine. Irradiances of the spectral lines corresponding to metallic species were constant throughout the test with respect to power level, indicating norma! engine wear. Similar results were obtained from the standard post firing engine analysis.

\section{References}

1. Cikanek, H.A., et al., "Space Shuttle Main Engine Plume Spectral Monitoring - Preliminary Results," AIAA Paper 87-1792, June 1987.

2. Linbaugh, C.C., et al., "Analys is of SSME Plune Images During Ground Testing," 1987 IAVNAF Propulsion Heeting. Vol. J. D.S. Eggelston and K.L. Strange, eds.. CPI.A-PLBL- $480-V 0 L-\bar{s}$. Chemical Propulsion Information Agency, Laurel. MD, 1987, pp. $215-223$ (Avail. NTIS, AD-B137172).

3. Powers. W.T., "Rocket Plume Spectrometry: A System Permitting Engine Condition Monitoring as tpplied to the Technology Test Bed Engine." Structural Integrity and Durability of Reusable Space Propulsion Systems, NASA CP-10030, 1989, pp. 127-135.

4. Powrs, M.T and Cikanek, H.A., "Analysis of UV-VIS Spectral Radiation From SSME Plume," Advinced Earth to Orbit Propulsion Technology 1983, Vol. 2, R.J. Richmond and S.T. Wu, eds., NASI CP-3012-VOL-2, 1988, pp. 595-611. 
5. Chenevert, D.J., "Combined Spectral and Video Monitoring of SSME Plumes," VASA John C. Stennis Space Center, Bay Saint Louis, MS. unpublished research.

6. Swaminathan, P.K., et al. "Calculation of Kinetic Data for Processes Leading to UV Signatures," AFOSR-89-0395TR, 1989 (Avail. NTIS, AD-A206656).

7. Young, S.J.. "Inversion of Plume Radiance and Absorptance Data for Temperature and Concentration," AFRPL-TR-78-60, 1978 (dvail. NTIS, AD-to68437).

8. Buchele, D.R., "Computer Program for Calculation of a Cas Temperature Profile by Infrared Emission: Absorption Spectroscopy," NASA-TM-73848, 1977 .

9. Reader, J., et al., "Wave lengths and Transition Probabilities for Atoms and Atomic lons," NSRDS-NBS-68, National Standard Reference Data System, Vational Bureau of Standards, 1980 (Avail. U.S.C.P.O., PB81-206120].
10. Pearse, R.W.B. and Gaydon, A.G., The Identification of Molecular Spectra, 3 rd ed. Wiley, New York, 1963.

11. Alkemade, C.J., et al., Metal Vapours in Flames, Pergamon Press, New York, 1982.

12. Ingle, J.D. and Crouch, S.R., Spectrochemical Analysis. Prentice Hall, Englewood Cliffs, NJ, 1988 .

13. Gonzalez, R.C. and Wintz, P.A, Digital Image Processing, Addison-Wesley, Reading, MA, 1977.

14. Press, W.H., et al., Numerical Recipes: The Art of Scientific Computing, Cambridge University Press, New York, 1986.

15. Marple, S.L., Jr., Digital Spectral Analysis with Applications, Prentice-Hall, Englewood Cliffs, N], 1987.

16. Cooley, J.W., Lewis, P.A.W., and Welch, P.D.,"The Fast Fourier Transform and its Applications," IEEE Transactions on Education. Vol. E-12, No. 1, Mar. 1969, pp. 27-34.

TABLE 1. Spectral Features in SSME Plume

\begin{tabular}{|c|c|c|}
\hline Species & Wavelengths $(\mathrm{nm})$ & Source \\
\hline Al & $308.22 / 309.27 / 394.40 / 396.15$ & Prosent in Structural Matorials \\
\hline $\mathrm{Ca}$ & $\begin{array}{l}422.67 / 443.50 / 445.48 / 445.58 / 534.95 / 558.88 \\
559.45 / 585.75 / 610.27 / 612.22 / 616.22 / 616.95 \\
643.91 / 644.98 / 646.26 / 647.17 / 649.38 / 649.97 \\
671.77 / 714.81 / 720.227732 .62\end{array}$ & Bearing Cages \\
\hline c & $\begin{array}{l}477.18 / 493.21 / 505.22 / 538.03 / 600.11 / 600.60 \\
601.32 / 601.48 / 658.76 / 711.32 / 711.52 / 711.70 \\
786.09 / 805.86\end{array}$ & Present in Structural Materiais \\
\hline $\mathrm{Cr}$ & $\begin{array}{l}357.87 / 359.35 / 360.53 / 396.34 / 425.44 / 427.48 \\
428.97 / 434.45 / 435.18 / 520.45 / 520.60 / 520.84\end{array}$ & Prosent in Structural Materiais \\
\hline$\infty$ & $\begin{array}{l}340.51 / 341.23 / 344.36 / 345.35 / 346.28 / 346.58 \\
347.40 / 350.23 / 350.63 / 352.69 / 352.98 / 356.94 \\
358.72 / 384.55 / 387.31 / 389.41 / 399.53\end{array}$ & Present in Structural Materials \\
\hline $\mathrm{Cu}$ & $\begin{array}{l}301.08 / 303.61 / 306.34 / 310.86 / 324.75 / 327.40 \\
330.80 / 353.04 / 406.26 / 465.11 / 510.55 / 515.32 \\
521.82 / 570.02 / 578.21 / 793.31 / 809.26\end{array}$ & MCC Liner, PB Baffles, LPOTP \\
\hline Au & $242.80 / 267.60 / 274.83 / 312.28 / 406.51 / 460.75$ & Brazing Malerials \\
\hline$H$ & $486.13 / 656.27 / 656.29$ & Propellant \\
\hline$F_{\theta}$ & $\begin{array}{l}344.06 / 358.12 / 371.99 / 373.49 / 373.71 / 374.56 \\
374.83 / 374.95 / 385.99 / 388.63 / 404.58 / 438.35\end{array}$ & Present in Structural Materials \\
\hline $\mathrm{Li}$ & $323.27 / 391.53 / 413.26 / 427.31 / 610.36 / 670.78$ & Dry Film Lubricant \\
\hline Mn & $403.08 / 403.31 / 403.45 / 404.14$ & Valves. Flex Joints \\
\hline Mo & $313.26 / 317.04 / 379.83 / 386.41 / 390.30 / 550.65$ & FPB/OPB Facoplates, Seals \\
\hline $\mathrm{Ni}$ & $\begin{array}{l}341.48 / 344.63 / 345.85 / 346.17 / 349.30 / 351.51 \\
352.45 / 356.64 / 361.94\end{array}$ & Present in Structural Materials \\
\hline$N$ & $\begin{array}{l}496.40 / 575.25 / 582.95 / 646.84 / 648.17 / 648.27 \\
648.38 / 648.48 / 740.62 / 42.36 / 744.23 / 746.83 \\
818.49 / 818.80 / 821.07 / 821.63 / 822.31 / 824.24\end{array}$ & Present in Atmosphere \\
\hline o & $\begin{array}{l}615.68 / 615.827700 .22 / 725.45 / 777.19 \pi 77.42 \\
\pi 77.54\end{array}$ & Propeliant \\
\hline k & $\begin{array}{l}404.41 / 404.72 / 578.24580 .18 / 581.22583 .19 \\
691.11 / 693.88 / 766.50 / 769.90\end{array}$ & Prosent in Propellants \\
\hline
\end{tabular}


TABLE 1. Continued

\begin{tabular}{|c|c|c|}
\hline Species & Wavelengths (nm) & Source \\
\hline Ag & $328.07 / 338.29 / 520.91 / 546.55$ & MCC, PB Baflles, LPOTP, Brazing \\
\hline $\mathrm{Na}$ & $\begin{array}{l}330.23 / 330.30 / 449.42 / 449.77 / 568.82 / 589.00 \\
589.59\end{array}$ & Present in Propoltants \\
\hline $\mathbf{S} r$ & $460.73 / 496.23 / 548.08 / 640.85 / 650.40 / 707.01$ & Present in Structural Matorials \\
\hline Ta & $\begin{array}{l}304.96 / 306.92 / 310.33 / 318.10 / 331.12 / 331.88 \\
337.15 / 360.74362 .66 / 364.21\end{array}$ & Prosent in Structurat Materials \\
\hline$\pi$ & $\begin{array}{l}319.20 / 319.99 / 334.19 / 335.46 / 337.14 / 363.55 \\
364.27 / 365.35 / 374.11 / 375.29 / 394.97 / 395.63 \\
395.82 / 398.18 / 398.98 / 399.86 / 430.11 / 430.59 \\
453.32 / 453.47 / 498.17 / 499.11 / 499.95 / 500.72 \\
501.42\end{array}$ & Present in Structural Materials \\
\hline$w$ & $400.88 / 407.44 / 429.46 / 430.21$ & LOX Posts, Turtines \\
\hline $\mathrm{CaO}$ & $547 \cdot 556$ & \\
\hline $\mathrm{CaOH}$ & $553 \cdot 557 / 620 \cdot 630$ & \\
\hline cro & $639-645$ & \\
\hline $\mathrm{CuH}$ & $425-435$ & \\
\hline $\mathrm{CuO}$ & $604-611 / 614-620$ & \\
\hline $\mathrm{CuOH}$ & $535-555 / 615-625$ & \\
\hline $\mathrm{F}_{\ominus \mathrm{O}}$ & $560-570 / 580-600 / 622-635 / 810-830$ & \\
\hline $\mathrm{F} \theta \mathrm{OH}$ & $353-358 / 363-368$ & \\
\hline $\mathrm{MnH}$ & $474-484 / 558 \cdot 586$ & \\
\hline $\mathrm{MnOH}$ & $350-400$ & \\
\hline $\mathrm{OH}$ & $305 \cdot 315$ & Combustion \\
\hline SrOH & $600-610 / 679 \cdot 685$ & \\
\hline Tro & $455 \cdot 495 / 515-525 / 545-555 / 610 \cdot 640$ & \\
\hline
\end{tabular}




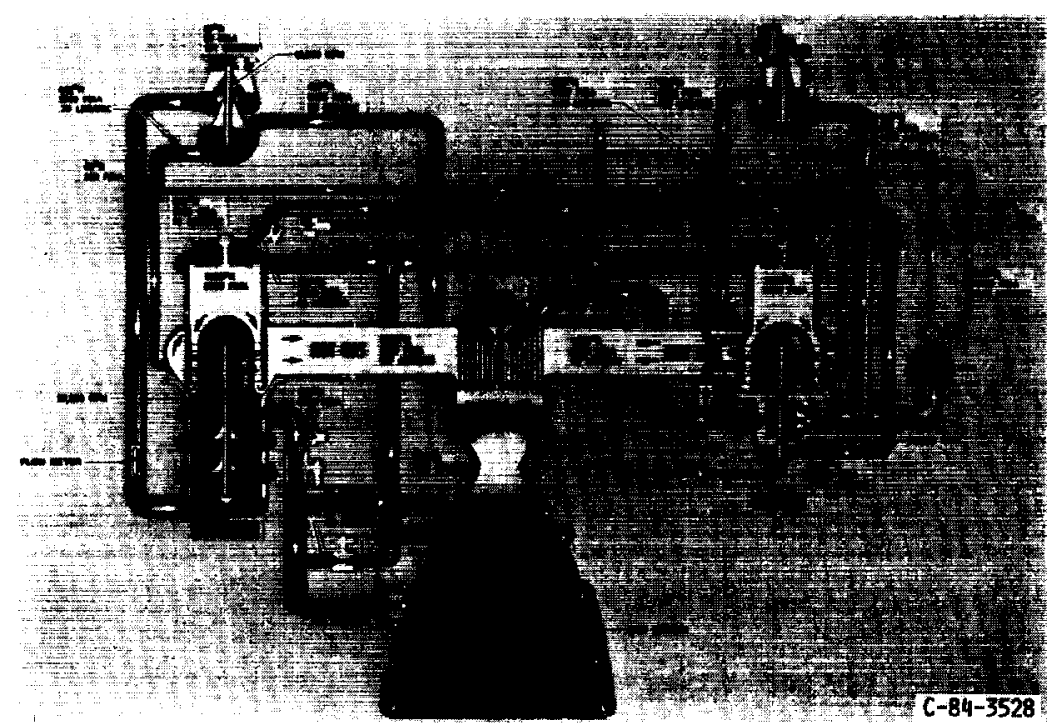

FIGLIRE 1. - SSME PROPELLANT FLOW PATHS.

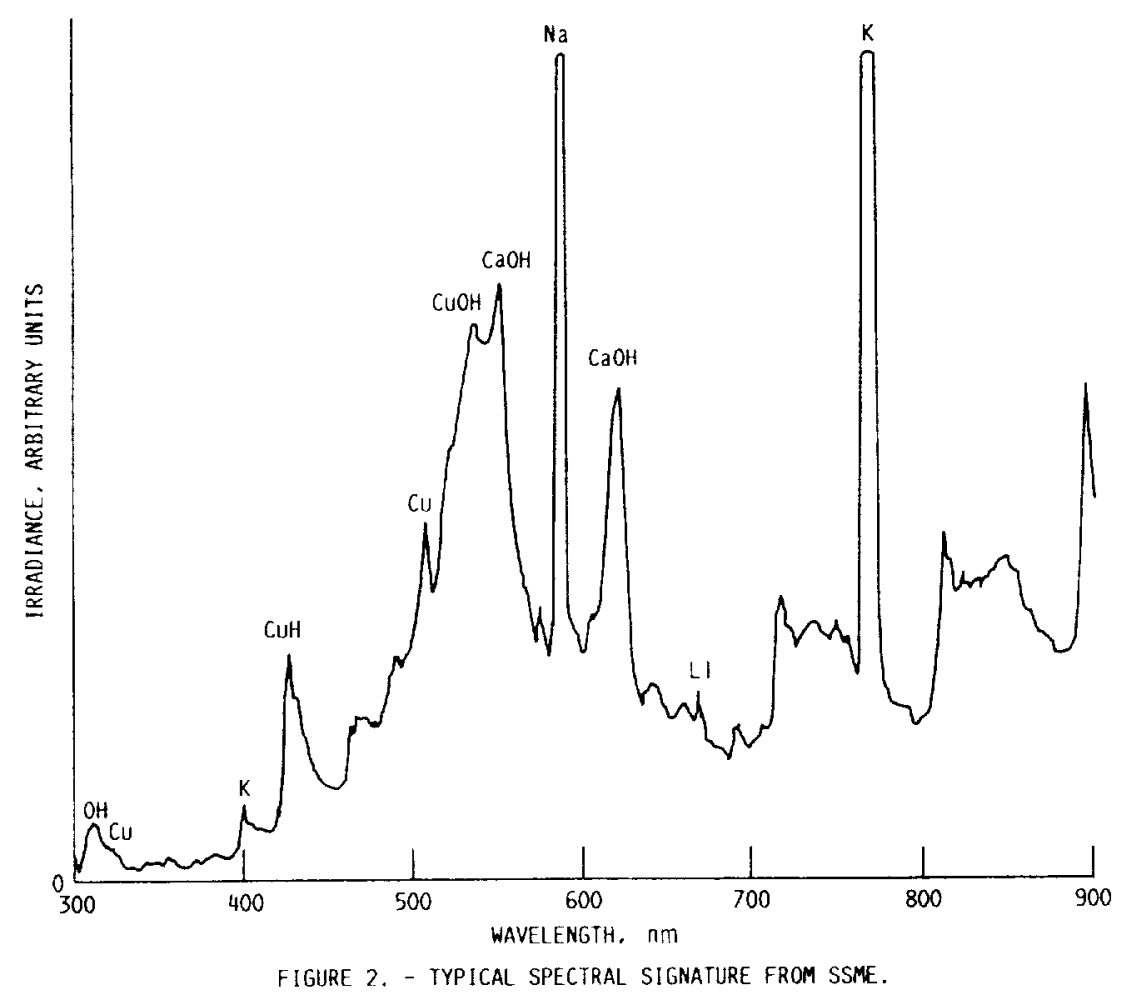




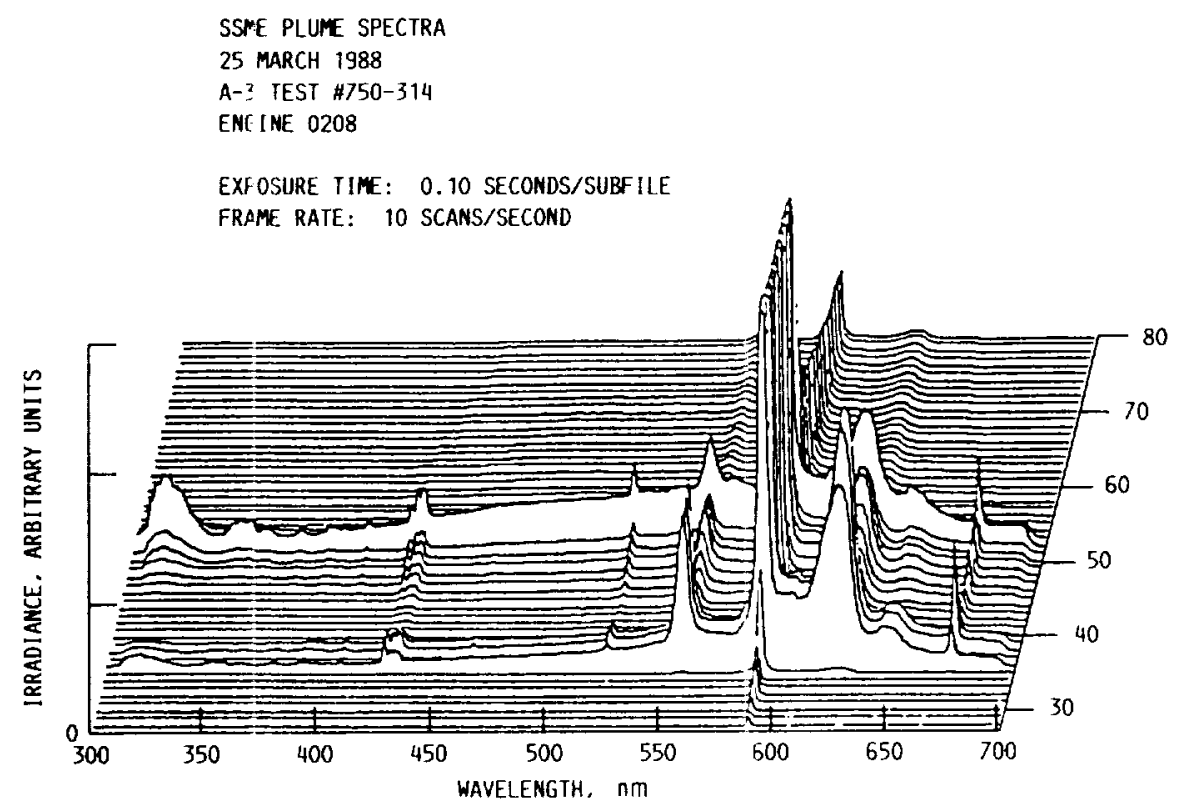

FIGURE 3. - TIME SERIES OF EMISSION SPPECIRA FROM SSME.

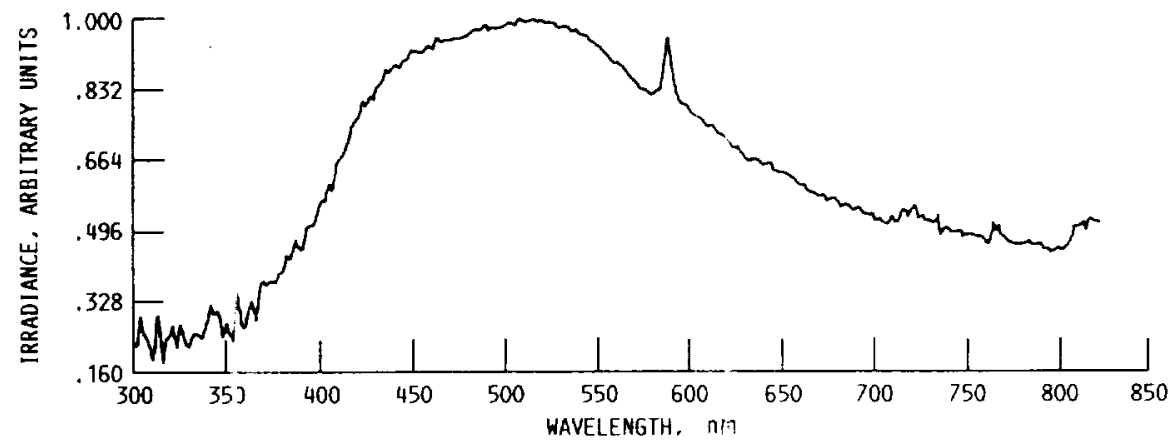

FIGIIRE 4. - SSME EMISSION SPECTRUM.

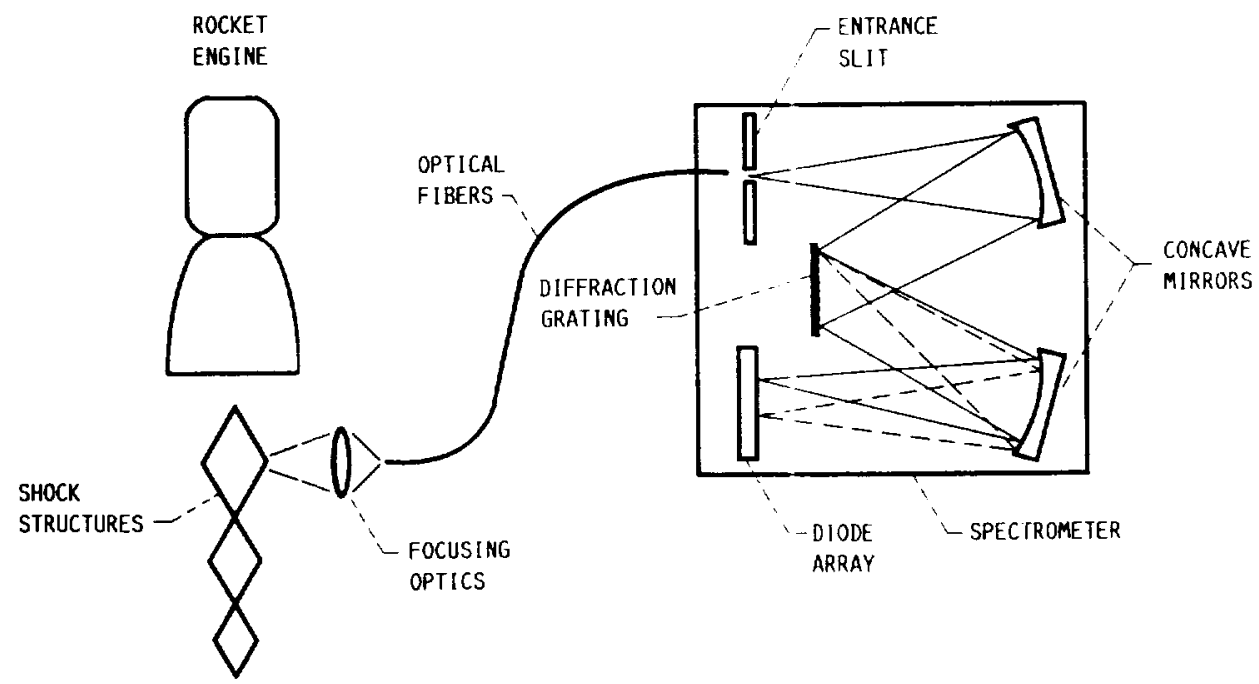

FIGURI. 5. - EMISSION SPECTROSCOPY OF ROCKET ENGINE EXHAUST PLUME. 


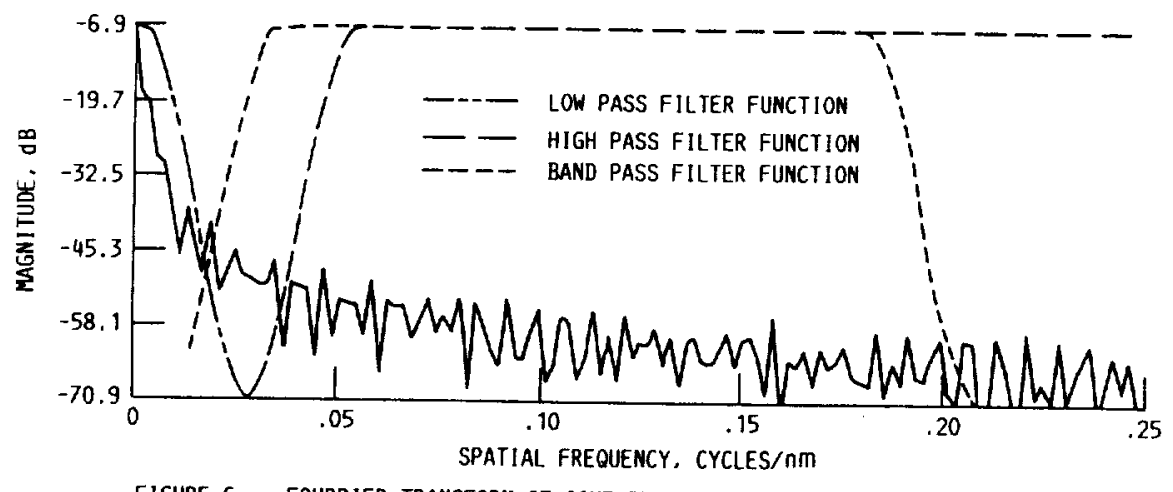

FIGURE 6. - FOURRIER TRAMSFORM OF SSME EMISSION SPECIRUM. DASHED LINES INDICATE THE VARIOUS FILTER FUNCTIONS.

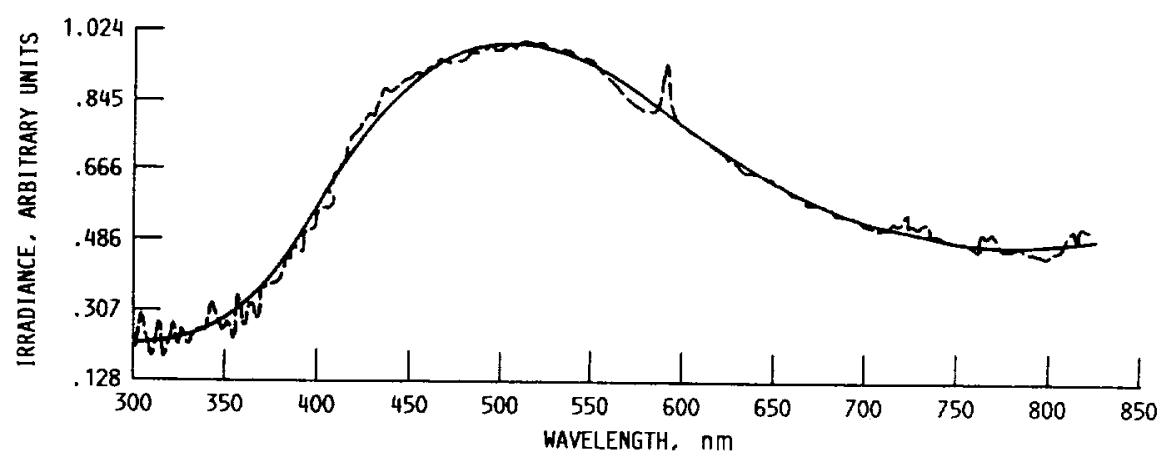

FIGURE 7. - EMISSION SPECTRUM AFTER LOW PASS FILTERIMG. THE SOLID LINE IS THE FILTERED SPECTRUM. AND THE DASHED LINE IS THE UNF ILTERED SPECTRUM.

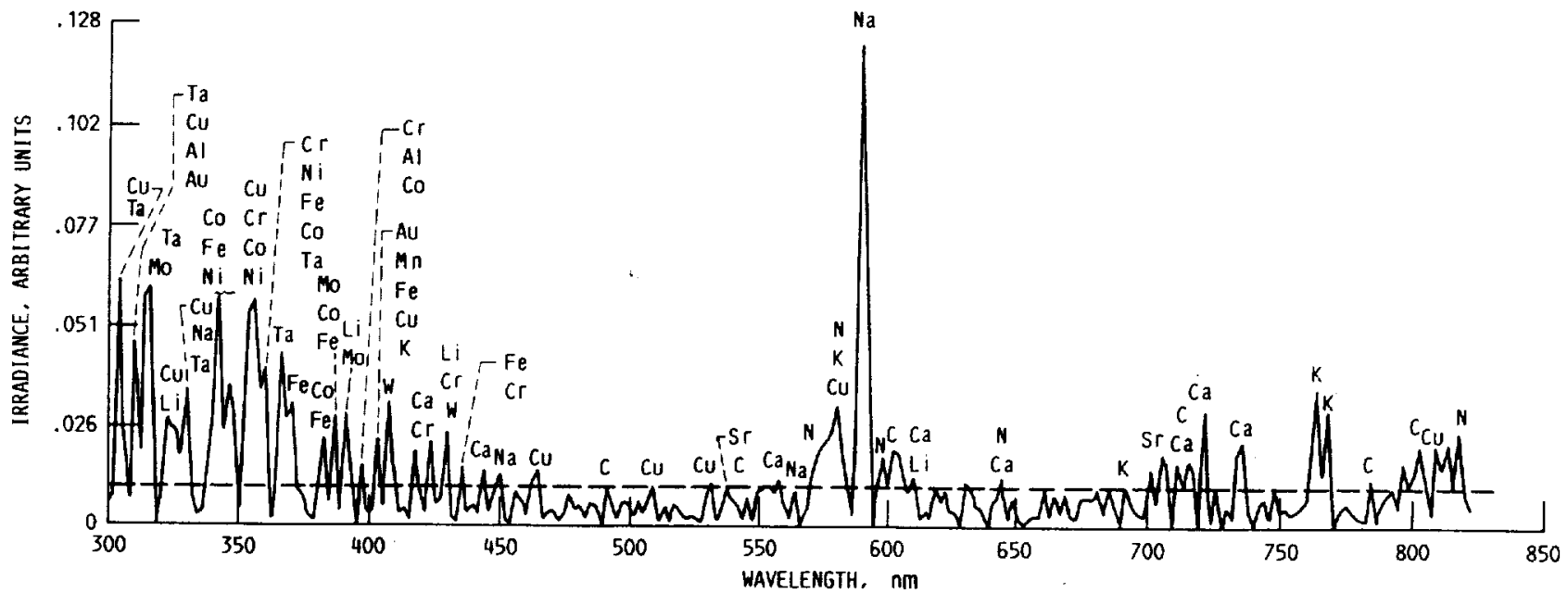

FIGURE 8. - EMISSION SPECTRUM AFTER HIGH PASS FILTERING. DASHED LINE INDICATES THE NOISE FLOOR. POOR SPECTROMETER RESOLUTION DID NOT ENABIE SEPARATION OF CLOSELY SPACED LINES: THEREFORE SOME LINES HAVE MULTIPLE LABLES. 


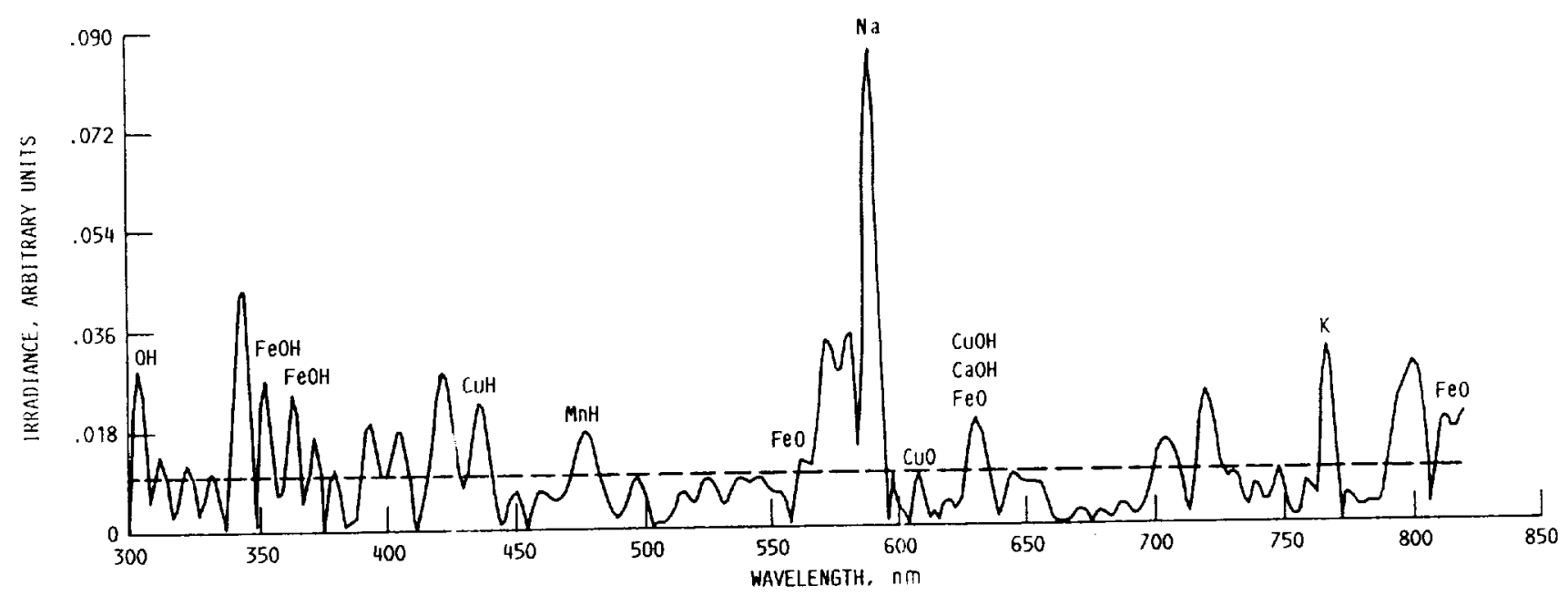

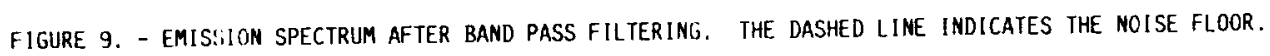




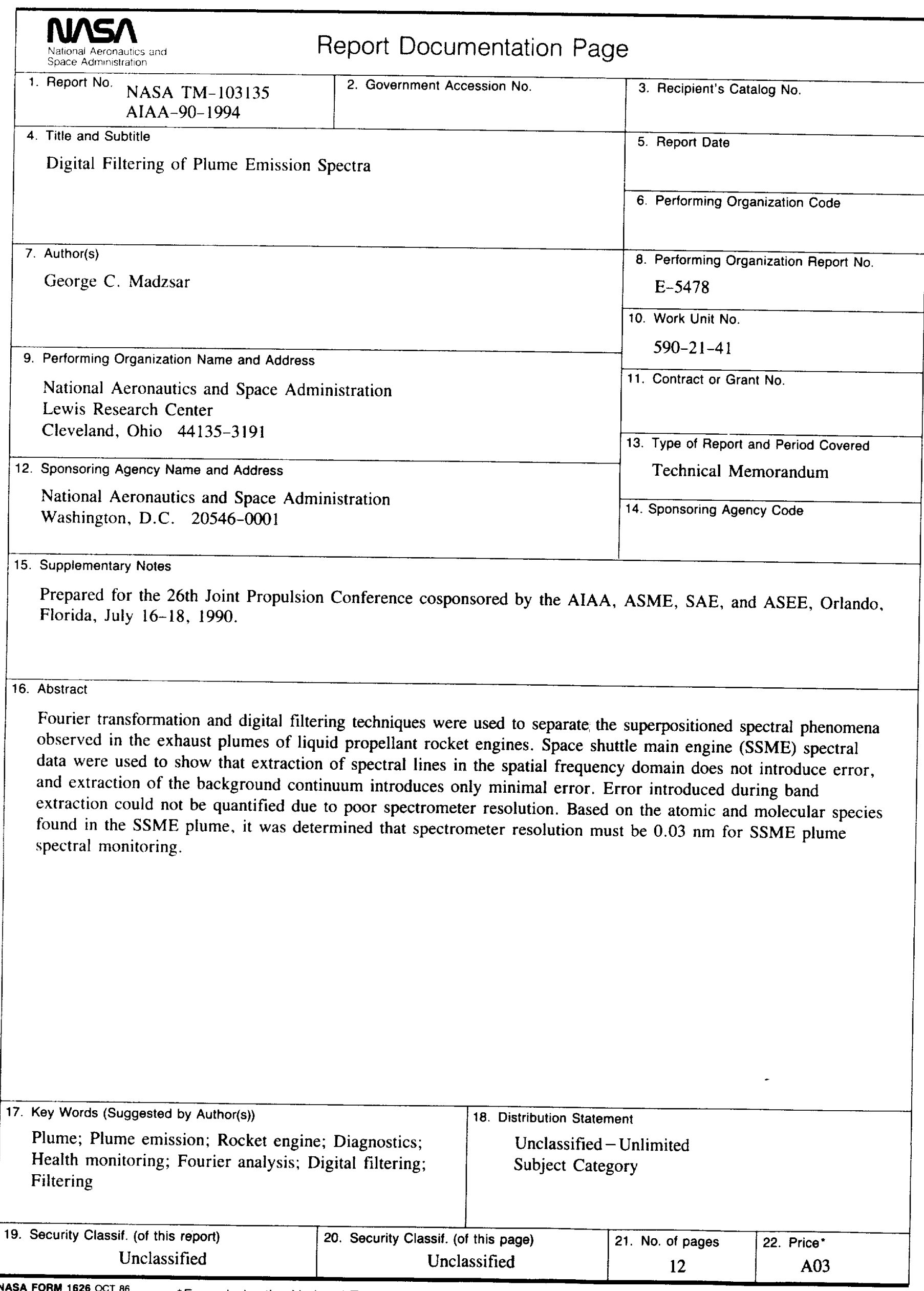

